\title{
Rabaska
}

Revue d'ethnologie de l'Amérique française

\section{Chaire de recherche du Canada en patrimoine religieux bâti (Université Laval)}

\section{Amélie Soulard et Tania Martin}

Volume 10, 2012

URI : https://id.erudit.org/iderudit/1013581ar

DOI : https://doi.org/10.7202/1013581ar

Aller au sommaire du numéro

Éditeur(s)

Société québécoise d'ethnologie

ISSN

1703-7433 (imprimé)

1916-7350 (numérique)

Découvrir la revue

Citer ce document

Soulard, A. \& Martin, T. (2012). Chaire de recherche du Canada en patrimoine religieux bâti (Université Laval). Rabaska, 10, 317-319.

https://doi.org/10.7202/1013581ar 


\section{QuÉBeC}

\section{Chaire de recherche du Canada en patrimoine religieux bâti}

École d'architecture, bureau 3235

Université Laval

Téléphone : (418) 656-2131 p. 2052

Québec (Québec) G1K 7P4

Télécopieur : (418) 656-2785

Courriel : Tania.Martin@arc.ulaval.ca

Toile : www.crcprb.chaire.ulaval.ca

Twitter:@CRC_PRB

\section{Journée d'étude sur le patrimoine et la géomatique}

Le 26 avril 2011, la journée d'étude portant sur la géomatique et le patrimoine bâti, organisée en collaboration avec le Centre de recherche en géomatique et la Ville de Québec, s'est déroulée à l'Université Laval, à Québec. L'événement a attiré plus d'une quarantaine d'intervenants des milieux professionnels, gouvernementaux et universitaires qui se sont réunis afin d'échanger des idées et des connaissances sur le thème "soutenir la gestion du patrimoine bâti à l'aide de la géomatique ». Cette journée d'étude fut également l'occasion de créer des liens dans le but d'harmoniser leurs efforts de gestion du patrimoine bâti et de soutenir les travaux futurs. La rencontre a pris la forme d'un atelier comportant deux séances de travail. Chacun des séances a débuté par une série de quatre courtes présentations (10 minutes) visant à faire état des travaux menés par des intervenants œuvrant dans le milieu de la sauvegarde, de l'interprétation et de la mise en valeur ainsi que la gestion du patrimoine bâti et de la géomatique.

Tandis que la première séance a permis d'aborder la question de l'intégration d'outils géomatiques aux processus décisionnels de manière à favoriser la concertation et le partage d'informations parmi divers acteurs, la deuxième séance a permis d'explorer le potentiel et les limites des outils géomatiques présentement utilisés dans la documentation, l'analyse, la représentation et la diffusion d'informations de nature variée propres au patrimoine bâti. À la suite des présentations, quatre tables de discussion ont été formées, chacune comprenant une dizaine de participants, un animateur et un secrétaire. Le temps alloué, environ une heure devait permettre aux participants d'échanger à partir de questions préparées à l'avance et des sujets abordés au moment des présentations. Enfin, chacune des séances a été complétée par une séance plénière où un rapporteur a résumé les discussions pour chacune des tables. Un rapport synthèse des discussions a été publié en version pdf sur le site de la Chaire.

La première édition de la journée d'étude sur le patrimoine et la géomatique a suscité un grand intérêt auprès des professionnels et universitaires œuvrant dans ces domaines. D'après les témoignages recueillis, cet événement a été une occasion riche d'apprentissage et de réflexion qui les aidera dans la poursuite de leurs travaux. Considérant ce franc succès, nous souhaitons reproduire la rencontre en repensant la formule pour permettre une plus large participation et l'approfondissement de thèmes plus circonscrits. 


\section{Formation}

Cours d'été Patrimoine bâti et paysages culturels in situ

Depuis 2007, la Chaire répond à l'invitation d'une communauté préoccupée par l'avenir du patrimoine bâti local et offre un cours intensif sur le terrain à des étudiants de divers horizons. L'approche pédagogique est basée sur l'expérimentation, l'immersion sur le terrain, les échanges multidisciplinaires entre étudiants, tout en étant encadré par une équipe d'experts. Après avoir eu lieu dans les communautés de Barachois, Malbay et Douglastown, la dernière édition s'est déroulée à Val-d'Espoir, une petite localité à l'intérieur des terres près de Percé reconnue comme l'une des plus dévitalisée de la région. La quinzaine d'étudiants a documenté et analysé les vestiges de l'école d'agriculture, dirigée par les Clercs de Saint-Viateur de 1938 à 1961, dans le but de proposer des pistes de mise en valeur. À l'issu des trois semaines de recherche, les étudiants ont livré leurs résultats à la communauté lors d'une journée de remue-méninges et ils ont produit une mini-exposition diffusée dans la communauté.

\section{Préparation du Forum international d'architecture vernaculaire}

En partenariat avec Patrimoine Gaspésie, la Chaire organise la première édition bilingue du Vernacular Architecture Forum qui se tiendra à Gaspé en juin 2013. La conceptualisation des circuits de visites guidées typique de cette manifestation donne l'occasion de rassembler, synthétiser et présenter les résultats des différentes recherches effectuées dans la région de Gaspé et de Percé par la Chaire depuis 2007 via les cours in situ et différents projets avec la communauté en 2010 (Bougainville, Pointe-Saint-Pierre et Val-d'Espoir) et 2011 (Anse-à-Griffon). Le thème développé pour le Forum est « le chassé-croisé de la religion et de l'économie dans les paysages culturels gaspésiens » permettant d'aborder les manifestations des visions du monde des groupes qui peuplent la pointe de la Gaspésie, les transformations historiques du cadre bâti des paysages et leur appropriation actuelle. La recherche se poursuit en 2012, la Chaire a rassemblé une équipe d'une douzaine de chercheurs (étudiants, diplômés et chercheurs universitaires) pour effectuer la recherche empirique complémentaire sur les sites inclus dans les circuits de visites, notamment un chafaud de Rivière-au-Renard, le sanctuaire en plein air de Pointe-Navarre et deux maisons préservées par Parc Canada à Forillon. L'ensemble du projet s'oriente vers une implication optimale des communautés et des acteurs locaux par la création de comités organisateurs et la tenue d'une journée d'étude sur les enjeux de mise en valeurs des paysages gaspésiens dans le programme du Forum (http://www.crcprb. chaire.ulaval.ca/fiav_vaf2013/).

\section{Mémoire d'Annie Pelletier - boursière France-Gagnon-Pratte, dépôt été 2012} «Espaces spirituels et communautaires de Mashteuiatsh. Lecture d'un paysage culturel ilnu ». Ce mémoire vise à comprendre les influences culturelles qui s'entrecroisent dans l'implantation, la construction et l'appropriation des espaces spirituels et communautaires de la communauté ilnue de Mashteuiatsh au Lac-Saint-Jean, Québec. Ces espaces sont l'indice d'une affirmation culturelle particulière en constante évolution et en réaction aux transformations qui ont entraîné et accompagné le passage d'un 
mode de vie nomade à la semi-sédentarité. Considérant qu'avec la sédentarisation les Ilnus ont adopté une pratique spirituelle hybride entre religions catholique et animiste traditionnelles, qu'en est-il de l'architecture ? Quelles ont été les transformations du bâti et du territoire et que révèlent-elles sur l'évolution culturelle de la communauté ? L'analyse se fonde sur l'approche des paysages culturels et de la morphologie urbaine. Elle superpose l'examen des archives écrites et graphiques, l'analyse visuelle et interprétative du cadre bâti et l'histoire orale des lieux détenus par les membres de la communauté. Cette approche met en évidence la « culturalisation», la permanence et les survivances de structures composant le paysage ilnu.

AmÉlie Soulard et TANia Martin

\section{Laboratoire de muséologie et d'ingénierie de la culture (LAMIC)}

Pavillon Casault, local 3645-Z Téléphone : (418) 656-2131, poste 3822 Université Laval

Québec (Québec) G1V 0A6

Courriel : Philippe.Dube@hst.ulaval.ca

Toile: www.lamic.ulaval.ca

Le LAMIC est un laboratoire de muséologie expérimentale qui peut compter, pour son fonctionnement, sur une petite équipe de chercheurs dont la direction est assumée par le professeur-chercheur Philippe Dubé et appuyée par un comité de direction et un comité scientifique composés de membres réguliers et associés du Laboratoire. La coordination des activités du LAMIC est assurée par la doctorante Dominique Gélinas. Le Comité de direction du LAmic est composé de Renée Bourassa, Lucie Daignault, Philippe Dubé, Dominique Gélinas et Nada Guzin-Lukic. Le comité scientifique est, quant à lui, constitué des professeurs Yves Bergeron, Philippe Dubé, Marie-Christiane Mathieu et Mathieu Rocheleau. Au total, le LAMIC rassemble plus d'une trentaine de personnes dont dix chercheurs, six doctorants et une dizaine de mémorants, sans compter les sept organisations partenaires. Cette infrastructure de recherche expérimentale a accueilli cette année une doctorante de France et deux stagiaires en maîtrise d'Haïti. La firme McG3D est intégrée au fonctionnement du LAMIC en mode de partenariat public/privé. Le LAMIC fait partie intégrante du CÉLAB - plate-forme réunissant les trois laboratoires du Centre interuniversitaire d'études sur les lettres, les arts et les traditions (CÉLAT), à savoir les Laboratoire d'archéologie de l'Université Laval (LAC), Laboratoire d'enquête ethnologique et de multimédia (LEEM) et le Laboratoire de muséologie et d'ingénierie de la culture (LAMIC). Ces derniers ne perdent pas leur structure individualisée, mais cette réunion permet le regroupement des forces technologiques de chacun selon les besoins ponctuels ou récurrents du CÉLAT. Par ailleurs, le LAMIC est rattaché à l'Itis de l'Université Laval et entretient des relations privilégiées de recherche avec le CERLIS, le CrÉAQ, l'IRHIS et la Centrale de Nantes en France.

Durant l'année universitaire 2011-2012 (de septembre à septembre), Philippe Dubé 